Cahiers d'études italiennes

\title{
La narrativa lesbica italiana nel contesto europeo. Note al margine
}

\section{Eleonora Pinzuti}

\section{OpenEdition}

\section{Journals}

Edizione digitale

URL: http://journals.openedition.org/cei/925

DOl: $10.4000 /$ cei.925

ISSN: 2260-779X

Editore

UGA Éditions/Université Grenoble Alpes

\section{Edizione cartacea}

Data di pubblicazione: 15 maggio 2008

Paginazione: 207-217

ISBN: 978-2-84310-121-2

ISSN: 1770-9571

Notizia bibliografica digitale

Eleonora Pinzuti, «La narrativa lesbica italiana nel contesto europeo. Note al margine », Cahiers d'études italiennes [Online], 7 | 2008, Messo online il 15 novembre 2009, consultato il 19 avril 2019 URL : http://journals.openedition.org/cei/925 ; DOI : 10.4000/cei.925 


\title{
LA NARRATIVA LESBICA ITALIANA NEL CONTESTO EUROPEO
}

\author{
NOTE AL MARGINE
}

\section{Eleonora Pinzuti}

Université de Florence

Una norma, nell'esperienza antropologica, non può essere originale. La regola non comincia ad essere regola se non agendo da regola, e questa funzione di correzione nasce dall'infrazione stessa.

Georges Canguilhem, Il normale e il patologico.

Quando mai t'ho chiesto l'eroismo dell'illusione?

Io ti ho chiesto se potevi trar gioia dalla realtà qual era [...]

Ti ho chiesto se io ero per te l'amore, come tu lo eri per me, in tutte le contingenze, da lontano come da vicino [...] separate da mille cose e pur unite in ogni respiro, salde, convinte di questa nostra unità, felici, felici, felici!

Sibilla Aleramo, Lettere d'amore a Lina.

Se l'oggetto della teoria narrativa non è il racconto ma la narratività, come relazione tra questa il senso, e il desiderio, e se un soggetto è «ingenerato» (come scrive Teresa De Lauretis $^{1^{*}}$ ) proprio dal suo inverarsi nel racconto, il dispiegarsi di certa tematica all'interno di una determinata letteratura dovrebbe rispecchiare, marxianamente, il livello antropologico della struttura sociale in quanto humus alle opere di finzione. Del resto il tema altro non è che una forma del topos, e il topos ingenera un livello di riferimento che, essendo simbolico, è strutturale e dunque risente delle dinamiche interne all'egemonia (gramscianamente intesa) del codice culturale : è il meccanismo generatore di testi di Lotman ${ }^{2}$.

Prendiamo quel che scrive Virginia Woolf in Una stanza tutta per sè : "Non ci sono uomini in questa stanza? Mi giurate che dietro quella tenda rossa non si nasconde Sir Charles Biron ? Mi assicurate di essere soltanto fra donne ? Allora vi posso dire che le prime parole che lessi furono : "Chloe voleva bene a Olivia"3 ». Queste parole, pronunciate di fronte alle

* Notes p. 215. 
ascoltatrici delle sue due conferenze sulle donne e il romanzo ${ }^{4}$, ci offrono subito l'occasione per ancorare il discorso dell'amore tra donne nell'ambito del culturalmente vietato : chiedersi se ci siano uomini ad ascoltare equivaleva infatti temere di infrangere il (comprensibile allora) confine fra ciò che ha diritto al racconto e ciò di cui invece è giusto tacere, secondo il canone dominante. L'amore fra donne (tanto vietato da essere stato nei vari codici penali spesso omesso perché inesistente) si presenta dunque come un discorso che necessita di essere decolonizzato da uno sguardo maschile che l'ha rappresentato secondo i feticci del proprio dettato. Il desiderio lesbico, descritto a tinte fosche da Charles Baudelaire, che nel suo Femmes Damnées pone in scena un rapporto quasi demoniaco, soggetto ai "travestimenti/travisamenti» del Balzac di La fille aux yeux d'or, gestito dalle descrizioni «iniziatiche» di De Sade (che vedeva nella sessualità fra donne solo "l'educazione sentimentale» per accedere al "vero sesso ", quello con i maschi), inventato da Pierre Louÿs nelle sue Chansons de Bilitis, non è dunque soggetto pronto a narrarsi, ma oggetto da sottrarre all'attenzione maschile per essere ri-creato attraverso il proprio bisogno di inverarsi nel narrato.

E peraltro evidente che la pubblicazione del racconto d'amore fra donne, rendendo "pubblico" un desiderio taciuto, lo rende pericoloso e inquietante (si potrebbe qui richiamare il «perturbante» freudiano) : del resto, citando Kaan, ben aveva ipostatizzato il problema Michel Foucault, quando scrive che "la causa della deviazione è l'immaginazione $e^{5}$ ", dunque proprio sull'immaginazione/immaginario, prima che divenga parola, si era imposto il silenzio. Questa sorta di « repressione addizionale ${ }^{6}$ ", cioè di repressione che agisce sul vissuto impedendone la vocalità tramite gli specifici interessi del dominio, si chiarisce meglio se si leggono le parole di Hayden White : «Se vediamo la narrazione e la narratività come strumenti attraverso i quali le esigenze contraddittorie dell'immaginario e del reale sono mediate, arbitrate o risolte in un discorso, cominciamo a comprendere insieme il fascino della narrazione e le ragioni per respingerla ${ }^{7}$ ». Ed è qui facile citare quanto dice Mario Mieli ( «L'esistenza del non-desiderio si riduce in gran parte all'esistenza di desiderio negato $\left.{ }^{8} »\right)$, che proprio in virtù della sua negazione, ancorandosi nel rimosso, trova un criterio di esistenza secondo le note teorie freudiane. Ma per quanto l'esistenza del rimosso agisca "sotto traccia ", in posizione tachigrafica rispetto alla grammatica e al discorso del racconto, questa condizione non può soddisfare il criterio della narrazione come forma espansa della soggettività : "l'epistemologia del chiuso» dunque (per citare Eve Kosofsky Sedgwick') ha necessariamente bisogno, in quanto epistemologia, delle forme di discettazione 
(dunque di liquidità simbolica) che le sono proprie. A questo proposito appare chiaro che la predisposizione innata al desiderio è costruzione e che l'intento di gestione normativa è volto al mantenimento dell'omeostasi sociale : «Le norme sociali guidano tale sviluppo verso la meta indicata, volta a volta, come giusta, reprimendo e selezionando i desideri e le loro espressioni verso ciò che viene stabilito "consensualmente" come degno di essere realizzato $^{10} »$.

Il rapporto dunque fra il detto e il taciuto si gioca in realtà seondo le categorie proprie del canone come langue all'interno della quale, saussurianamente, si innesta la parole, cioè la declinazione, lo scarto spitzeriano all'interno alla tradizione. Se, per dirla con Mireille Frauenrath, si analizza il rapporto biunivioco fra sujet donné e sujet traité ${ }^{11}$, è chiaro che il soggetto trattato (e le modalità paradigmatiche con cui questo viene trattato) risponde a contrario a tutta una serie di istanze immateriali che si concretano nella dimensione materiale, storica e paradigmatica del testo. Il testo dunque che tratta un tema non-trattato (anaforicamente, senza un trattato di genere che lo sostenga) si trova allora nella difficile situazione dell'irregolarità, che impone, per trovare una forma di comunicazione fra i codici, la necessaria ritraduzione del simbolico in un ordine meglio conosciuto. L'oggetto del discorso, essendo così eccentrico, permette dunque soltanto una soluzione di mediazione, che rispecchi in qualche modo una forma, limitata e limitante, dell'orizzonte di attesa. Il « corpo in figure» di Adriana Cavarero ${ }^{12}$, diviene allora necessariamente lo «scritto sul corpo" di Jeanette Winterson ${ }^{13}$, perché solo partendo da una scrittura della corporeità (quindi da un ordine di segni diverso da quello grammaticale proprio di un linguaggio normativo e normalizzante) si può perseguire un dettato che ricostruisca, dall'esterno, la storia di un desiderio che, perché desiderante, si fa tema e dunque narrazione.

La fuga quindi da una forma di "costruzionismo" del desiderio fra donne, se è iniziata fattivamente molto presto (e per una ricostruzione anche storiografica si veda l'insuperato studio di Daniela Danna ${ }^{14}$ ), ha risentito della funzione che a questo desiderio era stata data dalla cultura dominante, lottando per un processo di «decolonizzazione» che forse raccoglie oggi i primi sensibili risultati. Del resto, la difficoltà di una sub-cultura (intendendo con questo termine la difficoltà di accesso al paradigma dominante che tenta sempre una riduzione del simbolico a se stesso), fa sì che molta letteratura lesbica necessiti dell'aggettivo in quanto parte, caratteristica, connotato necessario alla tematizzazione specifica. Questo crea a sua volta un sapere minoritario, che nel rapporto fra canone e anticanone gioca le sue carte di autorappresentazione, ma che invera in sé i segni di un 
alfabeto che diverranno scarti stilistici e di riconoscimento (si pensi, appunto, a tutto il "saffismo» di una scrittrice come Renée Vivien ${ }^{15}$, a Violette Leduc ${ }^{16}$, Lidja Zinov'eva-Annibal ${ }^{17}$, che pur muovendosi fra stereotipie - da qui l'aggettivo connotato - misero in circolo modalità di riconoscimento, funzioni di racconto, e strategie finzionali che ebbero sicuramente un impatto sulle categotie rappresentative), cioè di trasmissione. Questo è ben argomentato da Iris Marion Young quando scrive :

Il gruppo definito dalla cultura dominante come deviante, come uno stereotipato Altro, è davvero culturalmente diverso dal gruppo dominante, perché la condizione di Alterità dà luogo a esperienze assolutamente specifiche e perché i gruppi culturalmente oppressi sono spesso socialmente segregati [...]. I membri di tali gruppi si comunicano gli uni con gli altri le specifiche esperienze e interpretazioni del mondo proprie del loro gruppo, elaborando e perpetuando così una loro cultura ${ }^{18}$.

Dunque per usare i sintagmi coniati da Giddens, la "coscienza pratica» della propria marginalità, attraverso il concetto di cultura si fa «coscienza discorsiva ${ }^{19}$ " proprio nel bisogno di narrativizzazione. La specificità del vissuto lesbico all'interno degli studi femministi ha trovato una sorta di collocazione categoriale nelle a-perimetrie dei queer studies, volti, com'è noto, a scardinare la fondazione di un sistema di tassonomie sul corpo tanto rigide quanto arbitrarie. Judith Butler a questo proposito chiarisce infatti come, all'interno delle filosofie femministe, la categoria onnicomprensiva del genere "donna ", che la Spivak definisce funzionale alla pratica politica, in realtà universalizzi una categoria ontologica nella quale molte donne non si riconoscono senza una ulteriore declinazione del termine :

questo atteggiamento ha creato un problema sia teorico che politico, e precisamente che un certo numero di donne appartenenti a diverse posizioni culturali si sono rifiutate di riconoscersi come "donne" nei termini formulati dalla teoria femminista, con il risultato che esse non rientrano nella categoria e vengono a trovarsi nella seguente alternativa : o non sono donne come avevano precedentemente ritenuto di essere, oppure la categoria riflette la posizione ristretta delle sue premesse teoriche, e, quindi, non è in grado di riconoscere l'intersezione del genere con la razza, la classe, l'etnia, l'età, la sessualità e le altre componenti che contribuiscono alla formazione della (non) identità culturale ${ }^{20}$.

Pare di sentir riecheggiare qui il «la lesbica non è una donna ${ }^{21}$ della Wittig, che destruttura il bourdiano campo del sapere aprendolo ad altre forme di intersecazione/costruzione delle identità. La Witting, autrice de Il corpo lesbico 22 , intendeva, con il suo oramai famoso epifonema, affermare che la lesbica non pertiene all'oggetto sociale della donna culturalmente intesa, e che invece è soggetto di una "pratica conoscitiva" che 
muta i rapporti sociali e le loro condizioni in posizione eccentrica rispetto alla normatività dell' istituzione eteropatriarcale.

Comunque, il termine stesso di identità (del resto refrattario, mobile, declinabile oramai in rivoli continui di auto-rivisitazioni), quando si diasistemizza in scrittura, comporta di necessità un riposizionamento all'interno della riflessione. Il tema infatti, e la sua rappresentazione, può declinarsi sia come auto-narrazione dell'esperire del sé (attraverso fome finzionali autobiografiche) sia come tema svincolato dall'identità soggettiva (che spesso comporta un elevato gradiente citazionale). Quel che però ci preme sottolineare è che, ci pare, mentre la tematica dell'amore fra donne, nelle letterature europee e occidentali del Novecento ha visto una sorta di fioritura all'interno del canone e delle modalità di rappresentazione (si pensi, per fare esempi corrivi, a The color purple di Alice Walker, ai romanzi di Vita Sackwille West e alla messe di narrazioni per le quali si rimanda all'utilissimo studio di Margherita Giacobino, Guerriere ermafrodite cortigiane ${ }^{23}$ ), la letteratura italiana, in questo si è mostrata meno ricca di esempi e narrazioni che siano state assimilate all'interno del canone, anche per la difficoltà che, a lungo, l'ambiente accademico ha mostrato nel recepirne le implicazioni e le aperture. Del resto, come osserva Francesco Polo :

[mentre] le grandi case editrici (Einaudi, Feltrinelli, Garzanti, Guanda, Mondadori, Adelphi) pubblicano Sibilla Aleramo, Alberto Arbasino, Dario Bellezza, Carlo Emilio Gadda, Pier Paolo Pasolini, Giuseppe Patroni Griffi, Giovanni Testori [...] Sul versante lesbico la situazione è, in parte, più desolante : bisogna aspettare gli anni '60 e '70 per leggere i romanzi (tradotti) di Radclyffe Hall (Dall'Oglio), Violette Leduc (Feltrinelli), Rita Mae Brown (Bompiani), Carson McCullers (Mondadori); e gli anni ' 80 per Willa Cather (La Tartaruga), Marina Cvetaeva (Guanda), Sheridan Le Fanu (Sellerio) ; romanzi e autrici d'importazione che si inseriscono in un sottobosco di saggi, racconti, poesie dell'amore tra donne confinato nelle autoproduzioni o microedizioni, mentre continuano a mancare grandi narratrici lesbiche italiane o, comunque, grandi storie lesbiche scritte da penne italiche ${ }^{24}$.

Facile qui sottolineare il sostrato culturale proprio della società in cui il prodotto letterario nasce e si sviluppa. Le ragioni di una parziale latitanza pertengono forse di più a una sorta di sociologia della narrazione : all'interno della stessa però, e dei prodotti che offre, possiamo comunque delineare alcune mappe utili al proseguimento della riflessione attraverso le modalità della stessa. Margherita Giacobino, in Orgoglio e privilegio. Viaggio eroico nella letteratura lesbica ${ }^{25}$, cita e analizza alcuni romanzi e racconti di Maria Rosa Cutrufelli, Complice il dubbio ${ }^{26}$, di Valeria Viganò, Il piroscafo olandese $e^{27}$, di Sara Zanghì, La cima della stella ${ }^{28}$, proponendo quest'ultimo come explicit dell'ideale silloge, poiché la Zanghì non pro- 
pone la sofferta differenza presente a vario titolo in Cutrufelli e Viganò. Alternando racconti che mettono in scena amori etero e lesbici, la Zanghì, secondo la Giacobino, fa sì che "la storia lesbica diventa storia di tutti e viceversa $^{29}$ ». Ora, se questo ragionamento può essere certo sostenuto, è chiaro che però evidenzia come la normalizzazione debba passare non tanto attraverso l'assimilazione del tessuto per farsi in qualche modo regola, ma che la compresenza di storie d'amore eterossessuale, "diluendo" il tema specifico nel più ampio soggetto d'amore, ne elide anche la focalizzazione e dunque gli effetti : dato che fa riflettere perché si parla di testi recentissimi.

Se prendiamo la raccolta di racconti a cura meritoria di Delia Vaccarello (meritoria appunto perché va a coprire una assenza assordante, se è permesso il banale sintagma) Principesse Azzurre $e^{30}$, edito da Mondadori (dunque una casa editrice di larghissima diffusione - e qui è necessario fare del paratesto genettiano, cioè soffermarsi sul supporto finanche editoriale che determina la diffusione) si legge nella prefazione :

L'amore tra donne non è più l'eccezione che non merita il canto. Un canto prima solitario, oggi a più voci. L'amore tra donne si può fantasticare, immaginare, intrecciare, rendere drammatico e lieve, virtuale e reale, si può narrare. Magia e favolosità, grazie al canto, diventano dimensione da vivere e non rifugio o maschera di delirio. La scrittura da qualche tempo si sta prendendo cura dell'amore tra donne. E lo nutre, lo tiene in vita ${ }^{31}$.

Il volume è uscito nel 2003, e basterebbe la data a creare frizione con il dettato : se la Vaccarello sente il bisogno, a fronte di una situazione evidentemente di non larga perimetria, di citare ancora la narrazione dell'amore fra donne come "maschera di delirio" (seppur in modo antifrastico, ma la necessaria precisazione è segno della necessità del disancoramento) e la dimensione temporale ("da qualche tempo ») viene ribadita, vuol dire che la soggettivizzazione del racconto lesbico non si è ancora del tutto emancipata dall'oggettivizzazione di una sottesa "diversità ». Il titolo stesso, che rimanderebbe a forme di riscrittura delle violenze proprie delle fiabe (si pensi a quelle di Angela Carter ne La camera di sangue $e^{32}$, volte a ricreare attraverso la dimensione morfologica proppiana delle fiabe altre funzioni che ingenerassero dimensioni diverse dall'obbligatorio orizzonte del "principe azzurro" come sogno d'amore obbligato per tutte le bambine), lo richiama in tralice come segnale quasi provenzale di presa di distanza. Ben diversa la seria a cura di Daniele Scalise, $M e n$ on $\mathrm{Men}^{33}$, che instaura subito un dettato ancorato alla corporeità senza ipotesti soggiacenti. Del resto, mentre nell'antologia a cura di Scalise gli scrittori firmano con il nome proprio, in Principesse Azzurre appaiono evidenti pseu- 
donimi (non molti, per la verità, ma sufficienti a rimandare a situazioni che rievocano non più il wildiano "amore che non dice il suo nome» ma chi non dice il suo nome dicendo l'amore), a traccia di una non liquida e pronta visibilità nominale del sé. Infatti, nella introduzione a Principesse Azzurre 2, Delia Vaccarello sente il bisogno di dire, a seguito di lettere di gratitudine delle lettrici : "Principesse azzurre è diventato un punto di riferimento, una speranza $"{ }^{34}$. Ora, il lemma è chiramente simbolo di un bisogno di immaginario, di una ricerca di autoriconoscimento, che ancora forse, nella letteratura italiana, non ha superato le dinamiche del canone e non è riuscita a imporsi non tanto come scrittura degna di lettura, ma come scrittura del continuo esperire umano che fa della tematizzazione soggetto di gnoseologia al pari dei topoi propri della tradizione (e dunque, archetipicamente, dell'esistenza).

La stessa necessità di nicknames è poi presente in un'altra antologia, Sabato sera a casa da no ${ }^{35}$, di recentissima pubblicazione, e sta ad indicare in fondo una non risolta assunzione di dettato che turba e lascia perplessi proprio per il rimando che sottende, e cioè che la visibilità della scrittura, là dove non sia ancorata alla visibilità personale, acquista un sapore domestico, privato. E se sono passati decenni dal famoso sintagma «il privato è politico ", è evidentemente a causa di una polis non così aperta alle differenze, che il privato rimane legato ad una dimensione di maschera. Questo rimanda dunque alle forme di rappresentazione ancora ancorate, almeno in parte, a stilemi non del tutto rielaborati e assunti come parti del soggetto sociale. È difficile infatti non citare un romanzo peraltro molto interessante come quello di Elena Stancanelli, Benzina ${ }^{36}$, dove le due protagoniste, Lenni e Stella, uccidono la madre di Lenni sullo sfondo di una pompa di benzina. Il noir lesbico, teorizzato da Paulina Palmer, ci permette qui di analizzare il genere del romanzo non tanto o non solo come genere popolare, ma per la ricaduta che il noir ha sul tessuto del romanzo. Le due ragazze commettono un omicidio uccidendo la madre di una di loro (sul simbolismo dello stesso è piuttosto facile per altro discettare) e dunque l'«eccentricità» del narrato (l'amore fra donne) viene facilmente sussunta e collegata all'atto omicida : in fondo la parodia delle "cattive ragazze ", l'evocazione di tanta letteratura on the road, rimandano però all'inquietante gesto che appunto lega la sessualità alla dimensione mortuaria, al disordine sentimentale, alla vischiosità (per sineddoche, quella della benzina) di un sentimento patologico. La lesbica assassina dunque (si pensi alle vampire o alle dimensioni mortuarie di primo Novecento : pensiamo a Guido Ceronetti e al suo La vera storia di Rosa Vercesi e della sua 
amica Vittoria $^{37}$ ), se qui può sussumersi in citazione e dunque in superamento, richiama però il topos con inquietante e serrata precisione.

Del resto, anche nell'ottica di una eventuale normalizzazione per la quale le lesbiche sono assassine o delinquenti come qualsiasi altro "gruppo" sociale, il dettato potrebbe sostenersi meglio se le dinamiche attraverso le quali si rappresenta il disordine (si pensi al celebre film Monster) fossero analizzate come impatto della disorganicità e non prevedibilità da parte del tessuto sociale ad un sentimento "imprevisto" (degenere), ma il fatto che il genere (in questo caso narrativo) del poliziesco lesbico abbia avuto un maggior successo che altre forme di racconto colloca di per sé l'espressione dello stesso in un sostrato che rimanda, comunque, al concetto di margine, rafforzandolo.

Melania Mazzucco, ne Il bacio della medusa ${ }^{38}$, mette in scena un femminile perturbante per nominalizzazione (Madlin detta Medusa e Norma, la donna borghese trascinata nei vortici della "perdizione» quando si allontana dalle dinamiche sociali), ancorando il racconto all'unhappy end proprio dei romanzi lesbici (e omosessuali in genere). Del resto in Lei così amata ${ }^{39}$, la funzione propria della biografia, legata com'è alla vicenda di Annemarie Schwarzenbach, non sottrae il dettato ad una lettura che friziona in tutto e per tutto con l'idea del lesbismo che circolava in Europa negli anni trenta, e che la Schwarzenbach per altro, suo malgrado, incarnò. Però, quello che qui ci preme sottolineare è che la narrazione biografica risente anch'essa del sistema modellizzante del narratore e ha funzioni ideologiche forti e pregnanti (si pensi all'exemplum agiografico). La vita dunque della Schwarzenbach rievoca per alcuni tratti un famoso romanzo, pubblicato nel 1928 e subito censurato, The well of loneliness ${ }^{40}$, di Radclyffe Hall, facendo rientrare in circolo il tema dell'androginia della lesbica e la sofferenza del vissuto, pur mediato da brani di intenso lirismo e profonda compartecipazione che inseriscono il lettore in una dimensione interna al narrato. Il gioco di specchi che si instaura dunque con la Schwarzenbach, non bruciandosi con la dimensione strettamente cronachistica o storica, si riverbera immediatamente in assunto contemporaneo. In Non dire il mio nome ${ }^{41}$ (quasi aposiopesi esistenziale e di un non esplicato vissuto se il nome ha funzione sociale e indica il primo criterio di esistenza), Paola Presciuttini narra un vissuto difficile, faticoso, quasi straniante per la recente uscita del romanzo.

Il finale è un finale mobile ( mi fermo qui per ora ${ }^{42} »$ ), non senza la straziante angoscia del racconto duro che proietta le ombre sull'intero vissuto della protagonista (si pensi alla scena sulla spiaggia, dove, nella vocalità orribilimente offensiva del maschio si risente in tralice il dettato gno- 
mico proprio delle forme di violenza fatte all'amore fra donne : non a caso infatti l'epigrafe che apre il libro recita : «Il calabrone, secondo le leggi dell'aerodinamica, non può volare : ma lui non conosce queste leggi, e vola ", ad indicare quanto il volo verso se stessi infranga le leggi del pensiero dominante).

Siamo comunque oramai lontane dal rischio che la narrazione (e dunque il desiderio) venga risommerso, come temeva Adrienne Rich. Vale la pena rileggere le sue riflessioni intorno ai significati da dare a quello che ancor oggi è un continente da decrittare e da storicizzare (vengono a mente le forme «resistenziali» dello "scrivere al buio» di bell hooks), proprio per fissare, senza violenza epistemica, una semiosfera dai margini e dalle perimetie tanto mobili e soggettive :

Esistenza lesbica sta ad indicare sia il riconoscimento della presenza storica delle lesbiche sia la nostra costante elaborazione del significato di tale esistenza. Per continuum lesbico intendo una serie di esperienze - sia nell'ambito della vita singola di ogni donna che attraverso la storia - in cui si manifesta l'interiorizzazione di una soggettività femminile e non solo il fatto che una donna abbia avuto o consciamente desiderato rapporti sessuali con un'altra donna ${ }^{43}$.

Se il sintagma «soggettività femminile» aprirebbe qui la necessaria discussione teorica sull'aggettivo, ci pare che queste parole stiano ancora ad indicare il percorso (storico, culturale, narrativo) che il corpo della lingua ha fatto, e deve ancora fare per definirsi e fissarsi in forme canoniche : infatti solo il topos permette la consecuzione e la trasmissione culturale. Il silenzio che ha avvolto la narrativa dei sentimenti d'amore fra donne, la Lettera aerea di Nicole Brossard ${ }^{44}$ che appunto non trovava terra, si deve ancorare al canone se vuole fecondare l'immaginario nella necessaria dialettica fra la propria continua specificità, le diverse forme in cui si esplica, e l'ascolto comune. Il corpo cancellato, e con lui il desiderio di narrazione per secoli sommersi, richiama ora le parole di Judith Butler :

The body that is on the couch is the same body that did the deed, but on the couch, the deed is relayed verbally ; the body acts again, but this time through the bodily act of speaking itself... what about the body ? It is the referent of the deed ; it is that whose activities are reported, relayed, communicated ${ }^{45}$.

\section{Notes}

1. Vedi T. De Lauretis, Sui generis. Scritti di teoria femminista, Milano, Feltrinelli, 1996 e T. De Lauretis, Pratica d'amore. Percorsi del desiderio perverso, Milano, La Tartaruga Edizioni, 1997.

2. Si vedano per le teorizzazioni intorno alla semiotica della cultura e ai meccanismi generatori di testi J. Lotman, Testo e conteso. Semiotica dell'arte e della cultura, Bari, Laterza, 1980 e J. Lotman, 


\section{Eleonora PinZuti}

Il testo e la storia. L'Evgenij Onegin di Puskin, Milano, Il Mulino, 1985.

3. V. Woolf, Una stanza tutta per sé, Milano, Il Saggiatore, 1993, p. 92.

4. «Ma, direte, noi le abbiamo chiesto di parlare delle donne e il romanzo - che c'entra il fatto di avere una stanza tutta per sé ?» V. Woolf, Ibid., p. 92. In realtà il saggio che darà il nome al libro si basa su due conferenze lette in due scuole universitarie femminili nel 1928 : la Arts Society di Newnham e la Odtaa di Girton, che avevano per tema, appunto, il rapporto fra le donne e il romanzo.

5. M. Foucault, Gli anormali, Milano, Feltrinelli, 2003, p. 249.

6. H. Marcuse, Eros e civiltà, Torino, Einaudi, 1977, p. 81.

7. H. White, The Value of Narrativity in the Representation of reality, in "Critical Inquiry ", 7, 1, autunno 1980, p. 8-9.

8. M. Mieli, Elementi di critica omosessuale, Milano, Feltrinelli, 2002, p. 174.

9. Cfr. E. Kosofsky Sedgwick Epistemology of the Closet, Berkeley, University of California Press, 1990.

10. P. Rigliano, Amori senza scandalo. Cosa vuol dire essere lesbica e gay, Milano, Feltrinelli, 2001, p.76.

11. M. Frauenrath, Le fils assassiné : l'influence d'un sujet donné sur la structure dramatique, München, Fink, 1974.

12. A. Cavarero, Corpo in figure. Filosofia e politica della corporeità, Milano, Feltrinelli, 1995.

13. J. Winterson, Scritto sul corpo, Milano, Mondadori, 1993.

14. D. Danna, Amiche, compagne, amanti. Storia dell'amore tra donne, Milano, Mondadori, 1994.

15. Renée Vivien è pseudonimo di Pauline Mary Tarn (1879-1909), autrice di un romanzo dal titolo Une femme m'apparut, dove con toni drammatici, del tutto volti a porre in scena un inevitabile maledettismo (appunto da femmes damnées) racconta della sua storia d'amore con Natalie Clifford Barney.

16. Ci si riferisce soprattutto a Thérèse et Isabelle, racconto di una passione lesbica, fortemente carnale, che esplode in un collegio femminile. Scritto nel 1955 uscirà in Italia, in edizione integrale per Baldini \& Castoldi solo nel 2002. La dimensione claustrale/collegiale era propria di un altro racconto, Olivia di Olivia (pseudonimo per Doroty Strachey), pubblicato anonimo nel 1949 in Inghilterra.

17. Autrice di un racconto dal titolo 33 mostri (Tridcat'-tri uroda, S. Petersburg, 1907), considerato il primo racconto lesbico della letteratura russa.

18. I. M. Young, Le politiche della differenza, Milano, Feltrinelli, 1996, p.77.

19. Vedi A. Giddens, Central Problems in Social Theory. Action, Structure, and Contradiction in Social Analysis, London, Palgrave Macmillan, 1979.

20. J. Butler, Genere, teoria femminista e discorso psicoanalitico, in Letteratura e femminismi, a c. di M. T. Chialant ed E. Rao, Napoli, Liguori Editore, 2000, p. 175.

21. Le Non Donne di Monique Wittig, intervento di Simonetta Spinelli al Convegno sulla Letteratura Lesbica, Casa Delle Letterature, Roma, giugno 2002.

22. Il corpo lesbico fu pubblicato in Italia nel 1976 (Roma, Edizioni delle donne).

23. M. Giacobino, Guerriere ermafrodite cortigiane. Percorsi trasgressivi della soggettività femminile in letteratura, Milano, Il Dito e La Luna, 2005.

24. F. Polo, L'editoria lesbica, gay, transessualel transgender, in We will survive! Lesbiche, gay e trans in Italia, a c. di P. Pedote e N. Poidimani, Milano, Mimesis Edizioni, 2007.

25. M. Giacobino, Orgoglio e privilegio. Viaggio eroico nella letteratura lesbica, Milano, Il dito e La Luna, 2003.

26. M. R. Cutrufelli, Complice il dubbio, Milano, Interno Giallo, 1992.

27. V. Viganò, Il piroscafo olandese, Milano, Feltrinelli, 1999.

28. S. Zanghì La cima della stella, Roma, Empiria, 1998.

29. M. Giacobino, op. cit., p. 115.

30. Principesse Azzurre. Racconti d'amore e di vita di donne tra donne, a c. di D. Vaccarello, Milano, Mondadori, 2003. Faranno seguito a questa prima raccolta, sempre per Mondadori e con la cura di D. Vaccarello, Principesse Azzurre 2 (2004), Principesse Azzurre 3 (2005), Principesse 
Azzurre crescono (2006), Principesse Azzurre da guardare (2007).

31. Ibid., p. 5. Corsivi nostri.

32. A. Carter, La camera di sangue, Milano, Feltrinelli, 1984.

33. D. Scalise, Men on men, Milano, Mondadori, 2002, a cui faranno seguito, sempre per Mondadori e sempre a cura di D. Scalise, Men on men 2 (2003) Men on men 3 (2004) Men on men 4 (2005) Men on men 5 (2006).

34. Principesse Azzurre 2. Racconti d'amore e di vita di donne tra donne, a c. di D. Vaccarello, Milano, Mondadori, 2004, p. 6. Corsivi nostri.

35. AA.VV., Sabaro sera a casa da noi, Forlì, Zoe Edizioni, 2007.

36. E. Stancanelli, Benzina, Torino, Einaudi, 1998.

37. G. Ceronetti, La vera storia di Rosa Vercesi e della sua amica Vittoria, Torino, Einaudi, 2000.

38. M. Mazzucco, Il bacio della medusa, Milano, Baldini \& Castoldi, 2006.

39. M. Mazzucco, Lei così amata, Milano, Rizzoli, 2000.

40. R. Hall, The well of loneliness, London, Jonathan Cape, 1928.

41. P. Presciuttini, Non dire il mio nome, Padova, Meridiano zero, 2004.

42. Ibid., p. 285.

43. A. Rich, Eterosessualità obbligatoria ed esistenza lesbica, in «Nuova DWF », 23-24, Roma, Coines, p. 26.

44. N. Brossard, La Lettre aérienne, Montréal, Editions Remue-Ménage, 1985.

45. J. Butler, Undoing Gender, New York, Routledge, 2004, p. 165. 